\title{
OPTIMIZATION OF THE WAVECAT WAVE ENERGY CONVERTER
}

\author{
H. Fernández ${ }^{1}$ G. Iglesias ${ }^{1}$ R. Carballo ${ }^{1}$ A.Castro ${ }^{1}$ M. Sánchez ${ }^{1}$ and F. Taveira-Pinto ${ }^{2}$
}

\begin{abstract}
The development of efficient, reliable Wave Energy Converters (WECs) is a prerequisite for wave energy to become a commercially viable energy source. Intensive research is currently under way on a number of WECs, among which WaveCat $($ - $-a$ new WEC recently patented by the University of Santiago de Compostela. In this sense, this paper describes the WaveCat concept and its ongoing development and optimization. WaveCat is a floating WEC intended for operation in intermediate water depths (50-100 m). Like a catamaran, it consists of two hulls - from which it derives its name. The difference with a conventional catamaran is that the hulls are not parallel but convergent; they are joined at the stern, forming a wedge in plan view. Physical model tests of a 1:30 model were conducted in a wave tank using both regular and irregular waves. In addition to the waves and overtopping rates, the model displacements were monitored using a non-intrusive system. The results of the physical model tests will be used to validate the 3D numerical model, which in turn will be used to optimize the design of WaveCat for best performance under a given set of wave conditions.
\end{abstract}

Keywords: Wave energy converter, Overtopping, Physical modeling, Numerical modeling, CFD.

\section{INTRODUCTION}

In order to reduce the emissions of greenhouse gases it is crucial to work along two lines. The first is to develop the already operational renewable energy sources, such as wind or photovoltaic energy. The second is to research and develop new energy sources (Iglesias et al., 2009). Among these, marine renewable energy have a great potential for development in Europe. The European Science Foundation estimates that "by 2050 Europe could source up to 50\% of its electricity needs from Marine Renewable Energy” (European Science Foundation, 2010). Marine Renewable Energy comprises offshore wind, wave energy, tidal currents, ocean currents, salinity gradient, thermal gradient and marine biomass (micro- and macro-algae). Among these, wave energy, offshore wind and tidal energy have arguably the greatest potential.

Two main issues must be resolved, however, for wave energy to become a fully established, commercially viable energy source. First, the wave resource must be assessed, for it presents significant spatial and temporal variations, as do other renewable resources. Second, efficient, reliable and low-impact Energy Converters (WECs) must be developed. This second aspect is, arguably, the greatest challenge. This paper deals with WaveCat, a recently patented WEC. Its objectives are: (i) to present the WaveCat concept; (ii) to describe the physical model tests conducted in a 3D wave tank and interpret its results; and (iii) to explain the numerical model that is currently under development.

\section{THE WaveCat CONCEPT}

WaveCat is a floating WEC intended for offshore deployment (water depths of 50-100 m). Offshore deployment has the advantage of a higher wave energy potential relative to onshore or nearshore locations - wave energy decreases as waves approach the shoreline. Another advantage of offshore WECs is their low visual impact.

The name WaveCat alludes to the fact that it is composed of two hulls, like a catamaran. Unlike a catamaran, however, these hulls are not parallel but convergent (Figure 1). The single-point mooring to a catenary-buoy allows WaveCat to swing as the wave direction changes, thereby ensuring the open side of the wedge always faces the waves (Figure 2). As waves propagate into the wedge, their height is enhanced by the convergence of the lateral boundaries (the hulls) until, eventually, they overtop the inner hull sides. Overtopping water is temporarily collected in on-deck tanks. The higher water level in the tanks relative to the sea level is taken advantage of to propel ultra-low head turbines as the water is drained back to sea.

A fundamental aspect in the design of any WEC-especially an offshore WEC-is its survivability, i.e. its ability to sustain heavy storm conditions. The survivability comprises three main aspects: design, engineering and construction. The design of WaveCat includes a number of elements aimed at survivability, most notably the possibility of varying the wedge angle between $120^{\circ}$ and $0^{\circ}$

\footnotetext{
${ }^{1}$ Group of Civil Engineering and Marine Energies, University of Santiago de Compostela, Campus Univ. sn, Lugo, 27002, Spain

${ }^{2}$ Faculty of Engineering, University of Porto, Rua Roberto Frias, Porto,Portugal
} 
according to the sea state. When a storm approaches, the angle is reduced to $0^{\circ}$, i.e. the wedge is closed, thereby transforming WaveCat into a monohull—similar to a conventional ship from the standpoint of seakeeping. Freeboard and draft are also variable. The hull length in the prototype is 175 $\mathrm{m}$.

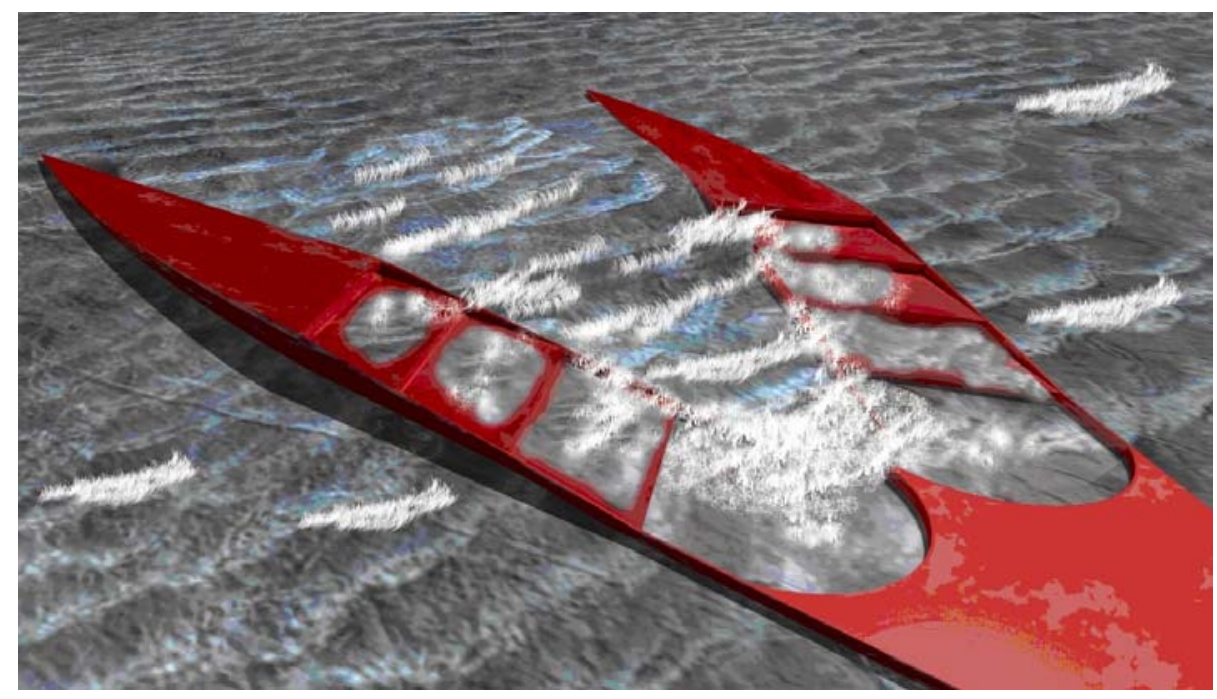

Figure 1. The WaveCat concept.

\section{OPTIMIZATION AND DEVELOPMENT}

The development and optimization of the WaveCat is being carried out by physical and numerical modeling. Concerning the physical modeling, the tests carried out in the FEUP wave tank are presented in Section 4, regarding the numerical modeling which is currently under development is described in Section 5.

\section{PHYSICAL MODELING}

The physical model was constructed in marine board and this represents the WaveCat at a 1:30 scale (Figure 2). The experimental campaign was developed in the wave tank of the Faculty of Engineering of the University of Porto, which main dimensions are $28 \times 12 \times 1.25 \mathrm{~m}$; this facility has a central pit of $4.2 \times 2 \times 1.5 \mathrm{~m}$ where the mooring system was anchored, (Figure 3). The waves were generated by means of a multi-element wavemaker formed by piston-type paddles. Six resistance wave gauges were employed to register the free surface position inside the wave tank, all of them were aligned with the central axis of the tank; another four wave gauges were used to register the water level time series in the model's reservoirs. The water depth was set to $0.9 \mathrm{~m}$ ( $2.4 \mathrm{~m}$ in the central pit) for all the tests. 


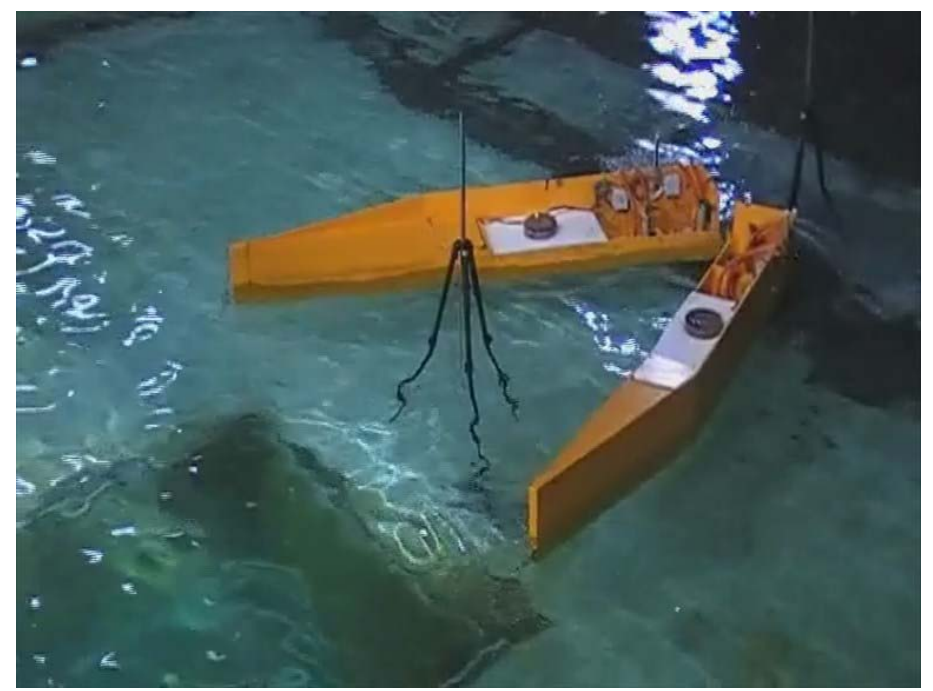

Figure 2. The physical model during an irregular wave test.

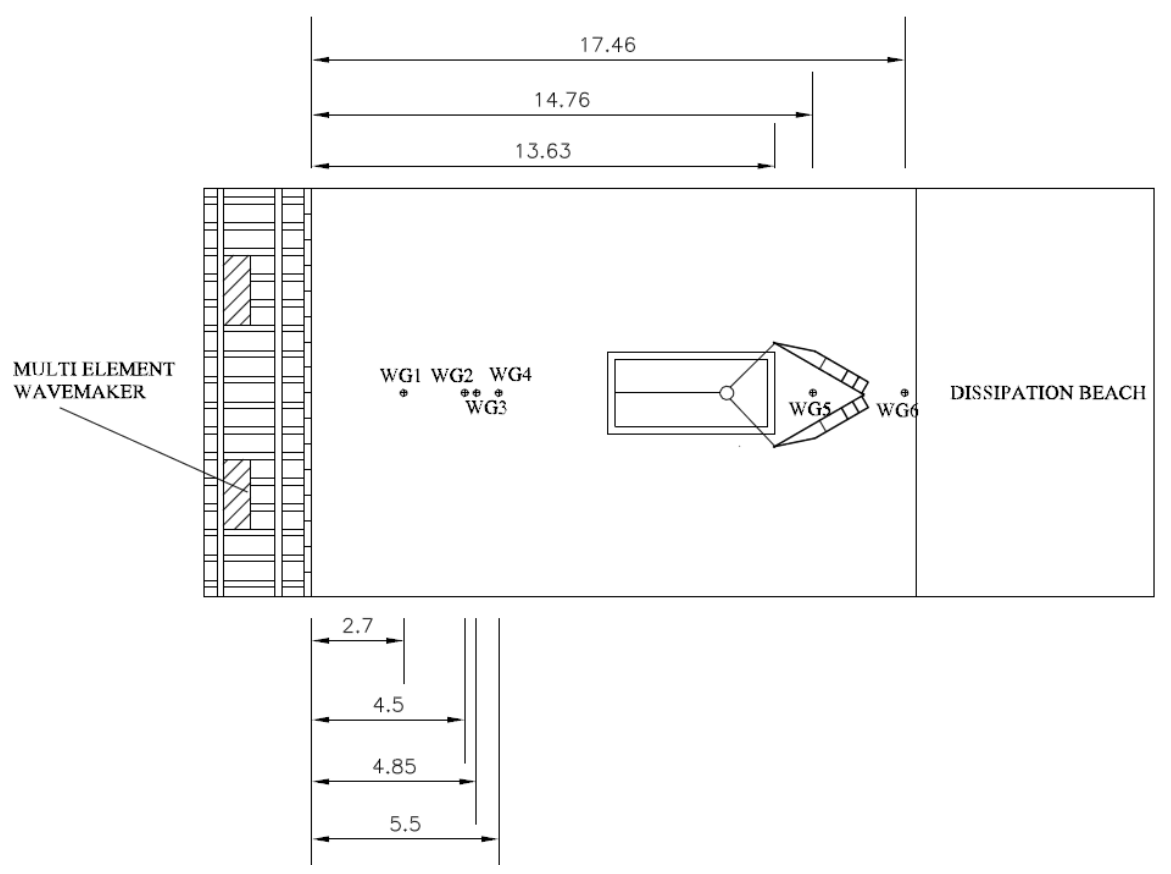

Figure 3. Experimental set-up used for the experimental campaign and wave tank sketch.

The experimental campaign consisted on 43 tests in total, 25 with regular waves and 18 with irregular waves. The wedge angle between the hulls varied between four values: $30^{\circ}, 45^{\circ}, 60^{\circ}$ and $90^{\circ}$. Three different freeboards were tested: $0.04 \mathrm{~m}, 0.09 \mathrm{~m}$ and $0.10 \mathrm{~m}$. Regular waves were in the ranges $\mathrm{H}=0.07-0.10 \mathrm{~m}$ and $\mathrm{T}=1.65-2.20 \mathrm{~s}$. Irregular waves varied between $\mathrm{Hs}=0.067-0.100 \mathrm{~m}$ and $\mathrm{Tp}=$ $1.83-2.20 \mathrm{~s}$. The testing program for the irregular wave tests are shown in Table 1 (in this case were conducted with the lowest freeboard). 
Table 1. Testing program for the irregular wave tests, parameters in model scale.

\begin{tabular}{|c|c|c|c|}
\hline Test case & $\alpha\left(^{\circ}\right)$ & $\mathrm{H}_{\mathrm{s}}(\mathrm{m})$ & $\mathrm{T}_{\mathrm{p}}(\mathrm{s})$ \\
\hline AA07_13 & 60 & 0.083 & 2.013 \\
\hline AA07_15 & 60 & 0.100 & 2.196 \\
\hline AB07_13 & 90 & 0.083 & 2.013 \\
\hline AB07_15 & 90 & 0.100 & 2.196 \\
\hline AD07_13 & 45 & 0.083 & 2.013 \\
\hline AD07_15 & 45 & 0.100 & 2.196 \\
\hline AE07_13 & 30 & 0.083 & 2.013 \\
\hline AE07_15 & 30 & 0.100 & 2.196 \\
\hline
\end{tabular}

The reservoirs placed in the model (two per hull) were equipped with a water level control system, formed by a bilge pump (working at constant flow rate) and a control system (Figure 4), this system starts when the water inside the reservoir reach a certain level and stops in a lower level. This system was employed to reach a desirable number of waves at each test The water level time series in two reservoir, \#3 and 4, during the test AB07_I5 is shown in (Figure 5). It can be observed that the reservoir \#3 experiments more overtopping than the reservoir \#4, this phenomenon occurs in all the tests.

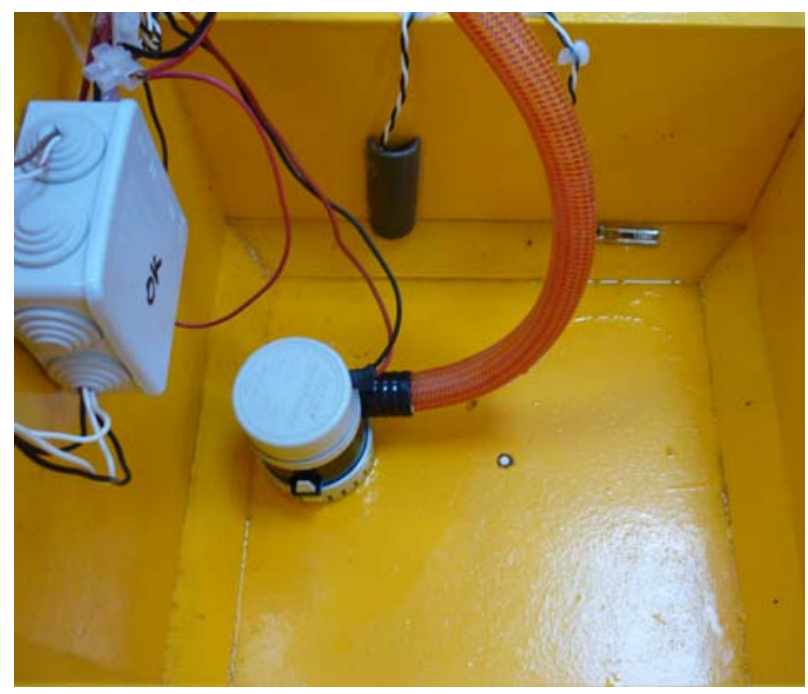

Figure 4. Water level control system formed by the bilge pump and the control system. 

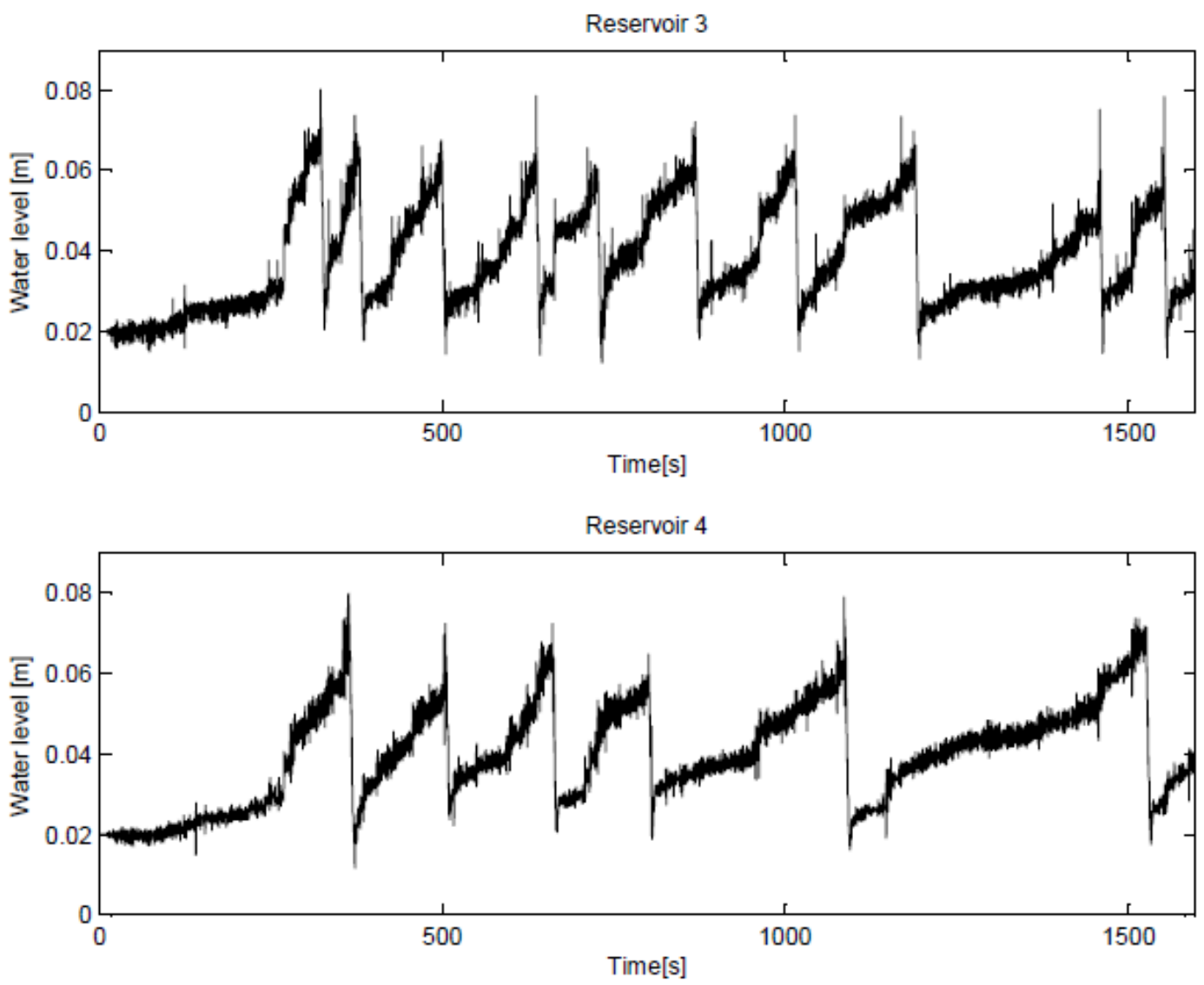

Figure 5. Water level time series corresponding to reservoir \#3 (above) and \#4 (below) during the test AB07_15.

In addition, a motion capture system was employed to register the main motions of the device. This system was formed by three infrared cameras placed strategically around the wave tank, five reflective marks placed in the model and an ad-hoc software implemented in a computer (Figure 6). One time the cameras detect the reflective marks (small spheres), the software creates a rigid body corresponding to the model which is monitored to register the main motions (heave, pitch and roll), the (Figure 7) shows a time series corresponding to the main motions of the model. 


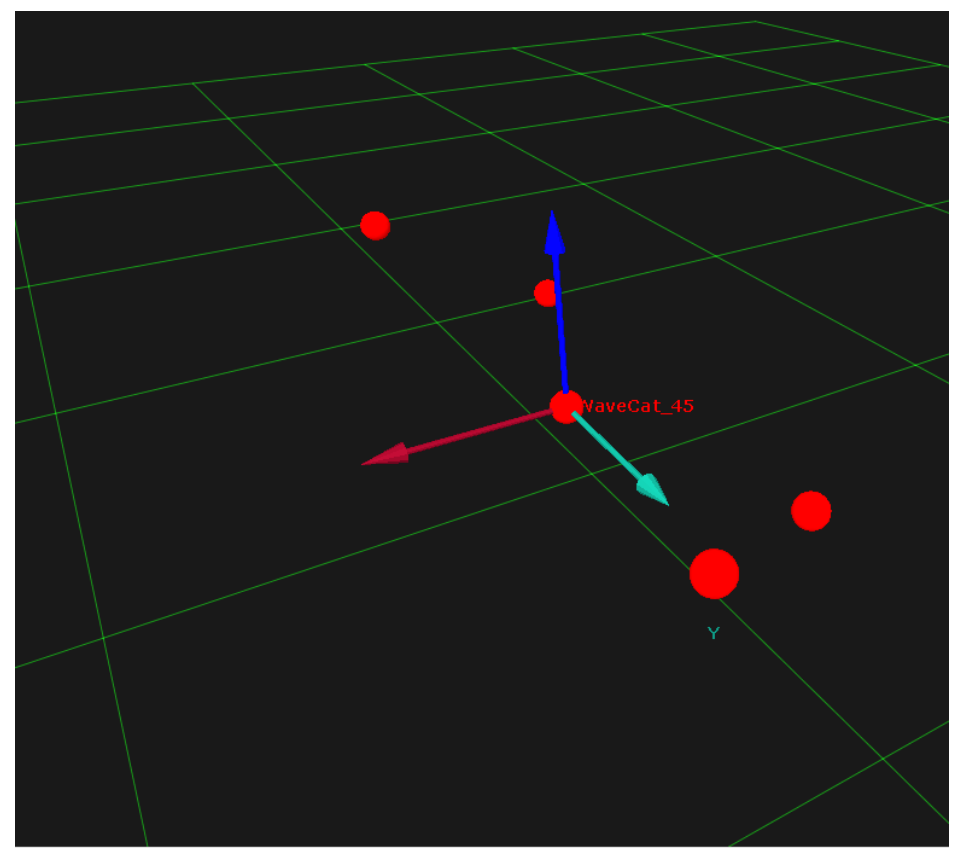

Figure 6. Reflective marks detected by the software.
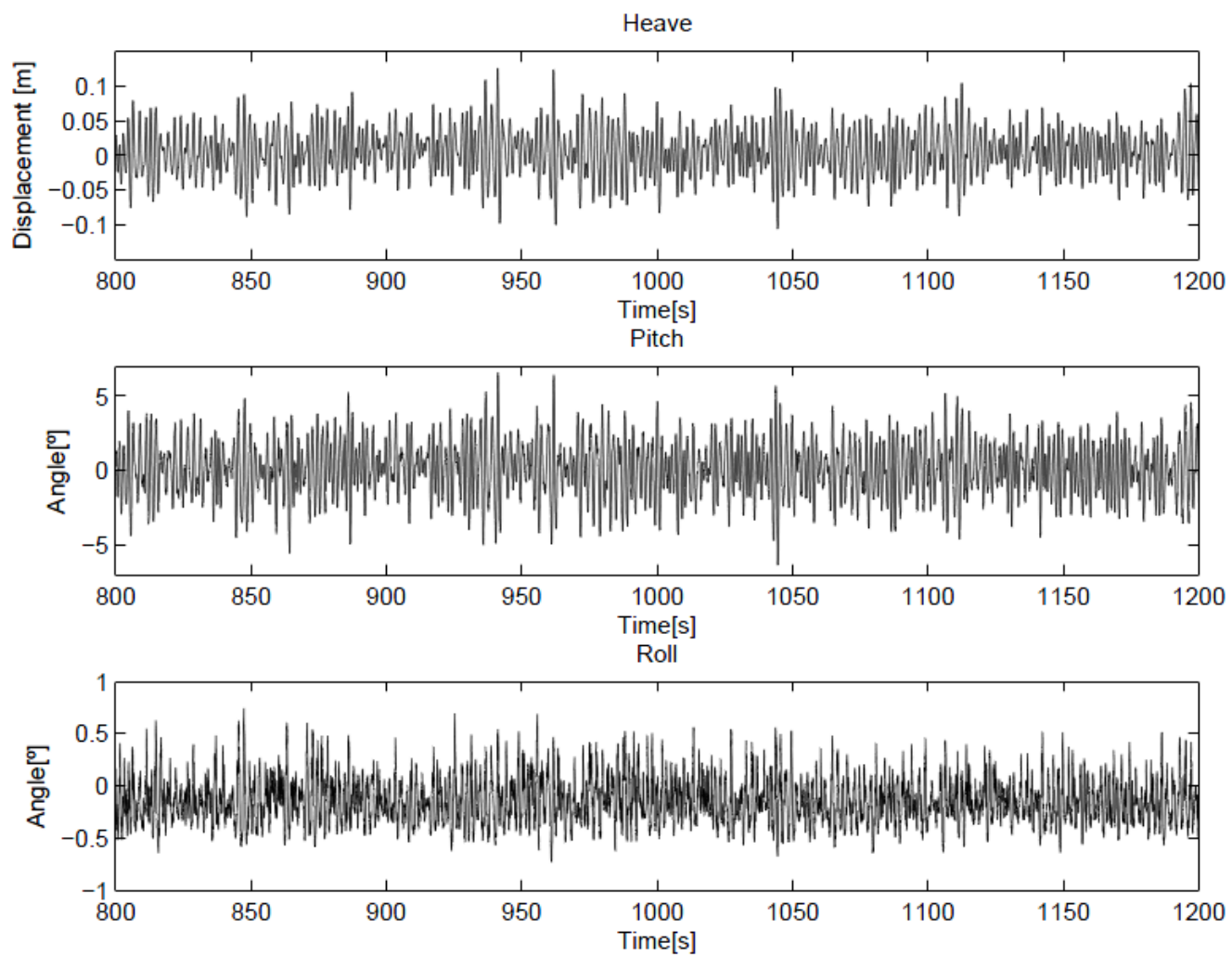

Figure 7. Time series of the main motions of the model (heave, pitch and roll) for the test AB07_15.

\section{NUMERICAL MODELING}

The first step with the numerical modeling was the validation of a 2D RANS-VOF model. This was successfully validated using the results from a 2D physical model carried out in the wave flume of the University of Santiago de Compostela. Nowadays a 3D numerical model is under development; this model solves the RANS (Reynolds Averaged Navier Stokes) equations with a volume-of -fluid 
approach to represent the free surface. This model simulates the response of the WaveCat model as a floating body interacting with the waves. This 3D numerical floating model is expected to be validated with the physical data presented in this paper. A frame of one simulation of this implemented model is shown in (Figure 8).

Solution Time $=29.2(s)$

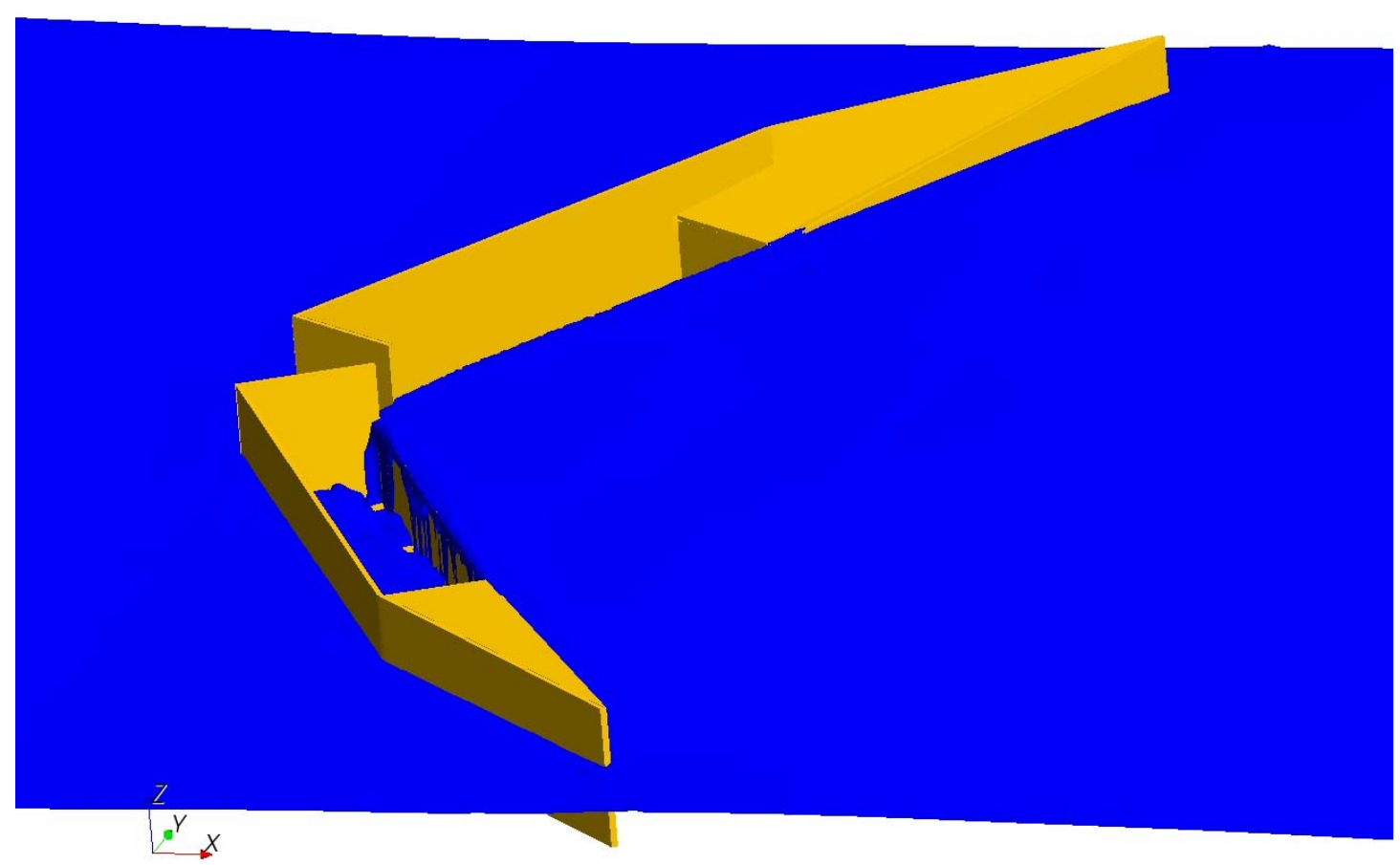

Figure 8. Image of the 3D numerical floating model.

\section{RESULTS AND CONCLUSIONS}

This paper describes the optimization and development work carried out and being conducted to make the WaveCat technology as a commercially viable system. In this sense, a 3D floating model of the WaveCat (built in marine board at 1:30 scale) was tested in a wave tank. The testing campaign consisted of 43 tests carried out with regular and irregular waves. In addition to the wave characteristics (wave height and wave period in case of regular waves and significant wave height and peak period in case of irregular waves), two fundamental model parameters were varied in the tests: wedge angle and freeboard. The motions of the model during the tests were recorded by means a nonintrusive system based on infrared cameras.

The tests results can be classified into three different levels. First, on a proof of concept level, these tests allowed to verify the WaveCat as a valid concept to convert the wave energy into electricity. As a second level concerns the design of the WaveCat and, in particular, the design of the water reservoirs. In this experimental campaign, the model has reservoirs with the same volume and with the same length along the hull side. It can be seen (Figure 5) that the fore reservoir, \#4, experiments lower overtopping than the aft reservoir, \#3, in this case for the test AD07_I5. This phenomenon was observed in all the tests of the experimental campaign, the fore reservoir collecting less water than aft reservoir. If this configuration is set in the future prototype, and a turbine-generator with same rate power is installed in all the reservoirs, the situation will be suboptimal. One way to solve this problem is changing the design of the reservoirs, increasing the volume of the fore reservoir in detriment of the 
volume of the aft reservoir, to balance the overtopping rates. Another way is maintaining the same volume in all the reservoirs and setting turbines with a greater rated power in aft reservoirs, because its larger overtopping rates. Nevertheless the first option seems to be more interesting, more research is necessary to take a final decision. Eventually, the third level of results regards on the recording of time series of overtopping rates and motions of the device during the tests; these will be used to calibrate and finally validate the 3D floating numerical model nowadays under development. One time this model will be validated, this will be used to optimize the design of the WaveCat under typical wave conditions of NW Spain (where the first prototype is expected to be deployed). After the optimization, the next step in the stage of development will be the construction of a demonstrator to make trials in the sea. Hence, the importance of the coupling of the physical and the numerical modeling in the work presented in this paper.

\section{REFERENCES}

G. Iglesias, M. López, R. Carballo, A. Castro, J.A. Fraguela, P. Frigaard, Wave energy potential in Galicia (NW Spain), Renewable Energy 34, 2009, pp. 2323-2333.

European Science Foundation, Marine Board Vision Document 2, 2010.

G. Iglesias, R. Carballo, Wave energy potential along the Death Coast (Spain), Energy 34, 2009, pp. 1963-1975.

G. Iglesias, R. Carballo, Wave energy resource in the Estaca de Bares area (Spain), Renewable Energy 35, 2010, pp. 1574-1584. 